\title{
Problems in Low Dimensional Contact Topology
}

\author{
John B. Etnyre and Lenhard L. Ng
}

During the 2001 Georgia International Topology Conference, two problem sessions were held concerning contact geometry. The first was run by Emmanuel Giroux and focused on problems in three dimensional contact topology; the second was run by John Etnyre and focused on Legendrian knots and contact homology. This article collects problems from the sessions, and adds some background. Sections 1 through 5 deal with three dimensional contact geometry, and Sections 6 through 10 deal with Legendrian knots.

We would like to thank the organizers of the Georgia Conference for running the problem sessions and encouraging us to write this problem list. We would also like to thank Ko Honda and Will Kazez, who took exceptional notes during the problem sessions, and Josh Sabloff, who compiled a list of some of the problems discussed here.

\section{Three Dimensional Contact Geometry}

In this section the reader is assumed to be familiar with the basic notions in contact geometry, see [31], and 3-manifold topology, see [49].

\section{Existence and types of contact structures}

QUESTION 1. Which 3-manifolds have tight contact structures, and what types of tight contact structures do they admit?

This question breaks down into several subquestions, but first note that a tight contact structure may be:

(1) weakly fillable (or weakly semi-fillable),

(2) strongly fillable (or strongly semi-fillable),

(3) holomorphically fillable (or holomorphically semi-fillable),

(4) universally tight, or

(5) virtually overtwisted.

Recall that a holomorphically fillable contact structure is also a strongly fillable contact structure, and strongly fillable implies weakly fillable. Moreover, a weakly

2000 Mathematics Subject Classification. Primary 57R17; secondary 53D35, 57M27.

Key words and phrases. Contact structure, tight, Legendrian knot, convex surface.

JE supported in part by NSF Grant \# DMS-9705949.

LN supported by the American Institute of Mathematics and NSF Grant \# DMS-9729992.

(C)2002 American Mathematical Society 
fillable contact structure is tight $[\mathbf{1 9}, \mathbf{4 8}]$. It is known that there are tight contact structures that are not weakly fillable [34] and weakly fillable contact structures that are not strongly fillable (the first such examples were found by Eliashberg [22] on the 3-torus, see also [15]). However, on homology spheres we know that weakly fillable structures must also be strongly fillable [69]. For a more thorough discussion of the notions of fillability and their relations, see [28].

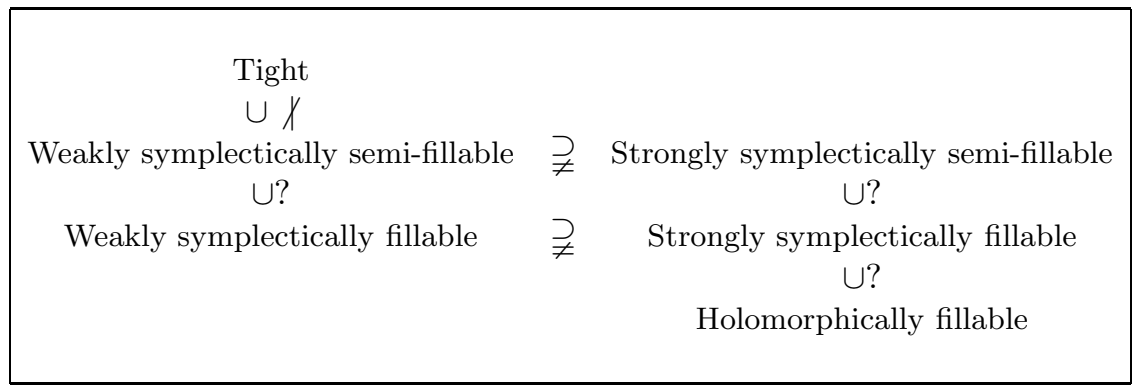

If a contact structure is fillable, it is interesting to consider the number and type of fillings. In particular:

Question 2. Do there exist only finitely many (strong or weak) fillings $\left(X^{4}, \omega\right)$ of a fixed $\left(M^{3}, \xi\right)$, up to blowup and blowdown?

Actually, it is not too hard to show there are always infinitely many strong fillings if we allow the fundamental group of $X$ to vary [74]. So it is most interesting to consider this question when $X$ is required to be simply connected.

The main technique used to show a contact structure is not fillable is SeibergWitten theory. This technique was initiated by Lisca [58] and only applies to manifolds with positive curvature. It would be exciting to find other methods for showing that a contact structure is not fillable.

\section{Question 3. How can one determine if a contact structure is fillable?}

It is obvious that universally tight and virtually overtwisted contact structures form disjoint sets, but it is not clear if all tight contact structures must fall into one of these sets. For any 3-manifold with a residually finite fundamental group, it is known (and not hard to prove) that any tight contact structure is either universally tight or virtually overtwisted. Recall that a group $G$ is residually finite if every nontrivial element of $G$ is in the complement of some finite index normal subgroup of $G$. Of course, it is conjectured that all 3-manifolds have residually finite fundamental groups.

There are no known connections between a tight structure being fillable (in any sense) and being universally tight or virtually overtwisted.

Question 4. Are universally tight contact structures fillable?

It is known that the converse is not true. An easier question might be the following.

QUESTION 5. If $\xi$ is a universally tight contact structure on $M$, is there some finite cover of $M$ in which the pulled-back contact structure is fillable?

We recall that the Poincaré homology sphere with the nonstandard orientation $-P$ does not admit a tight contact structure [32]. Moreover, any tight contact structure on the connected sum $M \# N$ can be decomposed into a tight contact 
structure on $M$ and a tight contact structure on $N$ [8]. Thus any 3-manifold of the form $M \#(-P)$ does not admit any tight contact structure, so when discussing existence of tight contact structures, we will always consider irreducible 3 -manifolds.

If one believes the conjectured picture of 3-manifolds coming from Thurston's Geometrization conjecture, then the main question breaks into two:

QUESTION 6. Do all hyperbolic manifolds admit a tight contact structure? What type of tight structures do they support?

QUESTION 7. Which Seifert fibered spaces admit tight contact structure? Which are fillable? Which are universally tight or virtually overtwisted?

Eliashberg and Thurston [26] have shown that a Reebless foliation may be perturbed into a tight (in fact, weakly semi-fillable and universally tight) contact structure. Moreover, Gabai [40] can construct such a foliation on any irreducible 3 -manifold with $b_{1} \geq 1$. Thus, when considering the above questions, one can focus on rational homology spheres, or consider strong fillability or virtually overtwisted contact structures.

Gompf [46] has constructed strongly fillable contact structures on many Seifert fibered spaces. Given the above results, here is our main concern: Let $\Sigma\left(\frac{\alpha_{1}}{\beta_{1}}, \frac{\alpha_{2}}{\beta_{2}}, \frac{\alpha_{3}}{\beta_{3}}\right)$ be a Seifert fibered space over $S^{2}$ with three singular fibers with invariants $\frac{\alpha_{1}}{\beta_{1}}, \frac{\alpha_{2}}{\beta_{2}}, \frac{\alpha_{3}}{\beta_{3}}$; then there is a strongly fillable contact structure on $\Sigma\left(\frac{\alpha_{1}}{\beta_{1}}, \frac{\alpha_{2}}{\beta_{2}}, \frac{\alpha_{3}}{\beta_{3}}\right)$ if $e_{0} \neq-1$, where $e_{0}=\sum_{i=1}^{3}\left\lfloor-\frac{\alpha_{i}}{\beta_{i}}\right\rfloor$ and $\lfloor x\rfloor$ is the greatest integer less than or equal to $x$. Moreover, Gompf can also construct fillable contact structures on many Seifert fibered spaces with $e_{0}=-1$; see $[46]$.

The only manifold currently known not to admit a tight contact structure is $-P$, which is a Seifert manifold over $S^{2}$ with invariants $-\frac{1}{2}, \frac{1}{3}, \frac{1}{5}$.

There are several other manifolds that do not admit weakly fillable contact structures. In $[\mathbf{5 8}, \mathbf{5 9}]$, Lisca has shown that the Seifert manifolds with invariants $\left(-\frac{1}{2}, \frac{1}{3}, \frac{1}{5}\right),\left(-\frac{1}{2}, \frac{1}{3}, \frac{1}{4}\right),\left(-\frac{1}{2}, \frac{1}{3}, \frac{1}{3}\right)$ do not admit weakly fillable contact structures. It would be very interesting to know:

QUESTION 8. Do the Seifert manifolds with invariants

$$
\left(-\frac{1}{2}, \frac{1}{3}, \frac{1}{5}\right),\left(-\frac{1}{2}, \frac{1}{3}, \frac{1}{4}\right),\left(-\frac{1}{2}, \frac{1}{3}, \frac{1}{3}\right)
$$

admit tight contact structures at all?

It is believed, at least by some, that these manifolds do admit tight contact structures. This result would be particularly interesting, because then these would be the first known manifolds admitting tight contact structures but not admitting any fillable structures.

As for hyperbolic manifolds, very little is known about the existence of tight contact structures. There are no known constructions of contact structures in terms of hyperbolic structures. It was once conjectured that all hyperbolic manifolds admit taut foliations. If this were true, we could perturb them into tight contact structures, but it has recently been shown that there are hyperbolic 3-manifolds without taut foliations $[\mathbf{6}, \mathbf{7 0}]$. It is still possible, however, that all hyperbolic manifolds admit a tight contact structure.

Another important question is:

QUESTION 9. Do all Haken manifolds admit a tight contact structure? 
Any manifold with $b_{1} \geq 1$ is known to be Haken, and from the work of EliashbergThurston [26] and Gabai [40], it is known that these manifolds admit semi-fillable, universally tight contact structures; see also [54] for a proof that avoids foliation theory. Thus our main interest here again is rational homology spheres.

\section{Uniqueness and classification}

Of course the main problem here is:

Problem 10. Classify tight contact structures on all 3-manifolds.

Currently contact structures are classified on the following manifolds:

(1) $S^{3}, S^{2} \times S^{1}[\mathbf{2 0}]$

(2) Lens spaces $[\mathbf{3 0}, \mathbf{4 3}, \mathbf{5 0}]$

(3) Torus bundles $[\mathbf{4 3}, \mathbf{5 1}]$

(4) Circle bundles $[\mathbf{4 4}, \mathbf{5 1}]$.

The classification is also known on some Seifert fibered spaces. For example, \# Tight $(\Sigma(2,3,5))=0$ or 1 , depending on orientation, where Tight $(M)$ denotes the set of tight contact structures up to isotopy. See [41] for further results on Seifert fibered spaces.

A specific problem whose resolution would have great importance for the classification of contact structures is:

Problem 11. Classify tight contact structures on $\Sigma \times[0,1]$, where $\Sigma$ is a surface of genus $g>1$.

In [55], Honda, Kazez, and Matić classify tight contact structures on $\Sigma \times[0,1]$, when the relative Euler class of the contact structure is extremal, $e(\xi)(\Sigma)= \pm(2 g-$ 2 ), and some mild conditions hold on the dividing curves. It seems much more difficult to understand the situation when the Euler class is not extremal.

This problem has relevance to the classification of tight contact structures on:

(1) surface bundles over circles,

(2) 3-manifolds with Haken decompositions, and

(3) general 3-manifolds.

The application of Problem 11 to surface bundles is obvious: given a tight contact structure on a surface bundle, split it along a convex fiber; the result is $\Sigma \times[0,1]$, and the dividing curves (or characteristic foliation) on the boundary components are related by the monodromy of the bundle. Now if one could normalize the dividing curves on a surface fiber, then one could apply the solution to Problem 11 to obtain an upper bound on the number of tight contact structures on the surface bundle. To get a lower bound, one could use Legendrian surgery and "state transition," see $[52]$.

A Haken decomposition of a 3-manifold is a way of successively cutting the manifold along incompressible surfaces until one arrives at a disjoint union of three-balls. Haken decompositions have been very useful in studying manifolds that support them $[\mathbf{4 0 , 7 5}]$. Honda, Kazez, and Matić $[\mathbf{5 4}]$ have used these decompositions to construct tight contact structures on manifolds with $b_{1} \leq 1$. The hardest step in this process is understanding the first cut. This would be greatly facilitated by answering Problem 11; it might then be possible to generalize the results of [54]. 
To study a general 3-manifold, one can use Heegaard decompositions. Any 3-manifold can be obtained by gluing two handlebodies together; such a representation of a 3-manifold is called a Heegaard decomposition. These decompositions have been very useful in studying 3-manifold topology (e.g., branched covers, Casson invariants, ...). So to understand tight contact structures on a 3-manifold, one might first want to consider:

Problem 12. Classify tight contact structures on handlebodies.

For handlebodies of genus 1 , this has been done $[\mathbf{4 3}, \mathbf{5 0}]$. Using techniques from [50], it is not hard to give an upper bound on the number of tight contact structures on a handlebody with fixed dividing curves on its boundary, but an actual classification seems difficult. If one could solve this problem, then to understand tight structures on an arbitrary 3-manifold, one would have to understand how to glue together tight structures on the handlebodies. Honda has developed a method called "state transition" for gluing tight contact structures together. In trying to implement this method, a solution to Problem 11 would be helpful.

Short of a complete classification, we can ask more specific questions. For example, we can start with the following motivational question.

QUESTION 13. Which manifolds admit only finitely many tight contact structures?

In $[\mathbf{9}, \mathbf{5 3}]$, it has been shown that any closed oriented irreducible toroidal 3-manifold admits infinitely many universally tight contact structures. Moreover, it has recently been announced, by Colin, Giroux, and Honda, that any closed oriented atoroidal 3-manifold admits only finitely many tight contact structures (for this statement, it is important to keep in mind that 0 is a finite number!). In light of these results, the above question, as stated, has been answered; however, there are, of course, many interesting refinements of this question. Foremost among them is:

QuESTION 14. Do all 3-manifolds admit only finitely many virtually overtwisted contact structures?

In $[\mathbf{9}, \mathbf{5 3}]$, the infinity of universally tight contact structures were constructed by first constructing one special universally tight contact structure, in which the incompressible torus $T$ has a neighborhood contactomorphic to $\left(T \times(-1,1), \xi_{1}\right)$, where

$$
\xi_{n}=\operatorname{ker}(\sin (2 \pi n z) d x+\cos (2 \pi n z) d y),
$$

$x, y$ are coordinates on $T$, and $z$ is the coordinate on $(-1,1)$. Then one obtains infinitely many tight contact structures by replacing $\left(T \times(-1,1), \xi_{1}\right)$ with $\left(T \times(-1,1), \xi_{n}\right)$. Intuitively, one is making the contact structure twist more near $T$. When doing this, one must prove that all the contact structures obtained are universally tight, and that they are different. To prove tightness, it is important that we begin with a special tight contact structure. To conclude that there are infinitely many different tight contact structures, one needs to know that the "twisting along $T$ is finite." This "twisting" is measured in $[\mathbf{9}, \mathbf{1 1}]$ using an invariant called the torsion of $T$ (see below), and in [53] using the twisting of various Legendrian knots transverse to $T$. In any event, it is not clear that a similar construction can be performed on virtually overtwisted contact structures.

The torsion of a torus $T$ in a contact manifold $(M, \xi)$ is the largest $n$ for which $\left(T \times(-1,1), \xi_{n}\right)$ can be contact embedded in $M$ so that $T \times\{0\}$ is isotopic to $T$; 
if no such $\xi_{n}$ can be embedded, the torsion is defined to be 0 . We would like to answer the following questions.

QUESTION 15. Are there only finitely many contact structures with minimal torsion?

QUESTION 16. Is torsion always finite in a tight contact structure?

For example, if we drop the hypothesis that the torus is normal in Colin's theorem [9], does an arbitrary tight $\xi$ have finite torsion? Note that for the infiniteness theorems of Colin and Honda-Kazez-Matić, which rely, more or less, on torsion being finite, the contact structures constructed are very special. We can also ask:

QUeSTION 17. How are torsion and fillability related?

In many specific examples (e.g., the tight contact structures on $T^{3}$ ), we know that only the tight contact structures with minimal torsion are strongly fillable, while the rest are only weakly fillable $[\mathbf{2 2}]$. Is this always the case? Specifically, we ask:

QUESTION 18. Can large torsion sometimes imply no filling?

When studying 3-manifolds one usually decomposes them in various ways. First, since any orientable 3-manifold has a unique prime decomposition, one usually restricts to irreducible manifolds. One may also do this when considering tight contact structures since for a connected sum $M_{1} \# M_{2}$, we know $\operatorname{Tight}\left(M_{1} \# M_{2}\right)=$ Tight $\left(M_{1}\right) \times \operatorname{Tight}\left(M_{2}\right)$; see [8]. Next, a 3-manifold is usually decomposed along incompressible tori. Specifically, given an irreducible orientable 3-manifold $M$, there is a collection of incompressible tori $T_{1}, \ldots, T_{k}$ such that the components of $M \backslash \cup T_{i}$ are atoroidal or Seifert fibered; moreover, this collection is unique if it is minimal with respect to this property. These tori give the Johannson-Shalen-Jaco (JSJ) decomposition of $M$.

QUESTION 19. Is there a contact JSJ decomposition?

More explicitly, suppose that $T_{1}, \ldots, T_{k}$ is a minimal system of tori given by the JSJ decomposition. Can we classify contact structures on $M$ if we have a classification on the pieces of $M \backslash\left(\cup T_{i}\right)$ ?

A related question is:

Question 20. Suppose two embeddings $\left(T^{2} \times I, \xi_{i}\right) \subset(M, \xi)$ are smoothly isotopic and maximal. Are they necessarily contact isotopic?

\section{Convex contact structures and open book decompositions}

A vector field $v$ on a contact manifold $(M, \xi)$ is called a contact vector field if the flow of $v$ preserves $\xi$. The contact structure $\xi$ is called convex if there is a gradient like contact vector field on $M$. Recently Giroux has proved (using ideas from [42]) that any contact structure on a closed 3-manifold is convex. This gives us another tool to study contact structures.

QUESTION 21. How are properties like fillability and virtual overtwistedness reflected in convex vector fields associated to a given contact structure?

For example:

QUestion 22. Does the dynamics of the flow of a contact vector field say anything about the contact structure? 
Giroux [45] has provided an interesting approach to Question 21. Recall that an open book decomposition of a closed 3-manifold $M$ is a fibration of the complement of a link $L$, called the binding of the open book. If $F$ is the fiber of the fibration of $M \backslash L$, then $M \backslash L$ is obtained from $F \times[0,1]$ by gluing $F \times\{1\}$ to $F \times\{0\}$ by a diffeomorphism $\phi: F \rightarrow F$ called the monodromy. Following Thurston and Winkelnkemper [76], one can find a 1 -form $\lambda$ on $F$ such that $d \lambda$ is a volume form on $F$. Then $\alpha=d t+t \cdot \lambda+(1-t) \cdot \phi^{*} \lambda, t \in[0,1]$, is a contact form on $F \times[0,1]$ that descends to a contact form on $M \backslash L$, and it is not hard to extend $\alpha$ over $M$. The contact structure $\xi=\operatorname{ker} \alpha$ is said to be adapted to the open book decomposition. Giroux has shown how to construct a contact vector field on $(M, \xi)$ that is transverse to the fibers of the open book. Thus from an open book decomposition, one gets an adapted convex contact structure. Moreover, given a convex contact structure, Giroux can construct an open book decomposition to which the contact structure is adapted. Hence there is a one-to-one correspondence between convex contact structures and open book decompositions.

Generalizing work of Loi and Piergallini $[\mathbf{6 2}]$ (see also $[\mathbf{2 , ~ 6 3 , ~ 6 6 ] ) , ~ G i r o u x ~ c a n ~}$ show that a contact structure is Stein fillable if and only if it is adapted to an open book decomposition whose monodromy is a product of positive Dehn twists. Thus we can refine Question 21 to:

QUESTION 23. What conditions on the link or monodromy map of an open book decomposition imply that the adapted contact structure is tight? (Weakly or strongly) symplectically fillable? Universally tight? Virtually overtwisted?

Fix $M$ and a contact structure $\xi$. Let $\mathcal{B}(\xi)$ be the set of all open book decompositions to which $\xi$ is adapted. Let $g(\xi)=\min \{\operatorname{genus}(F)\}$, where the minimum is taken over all fibers $F$ of fibrations in $\mathcal{B}(\xi)$.

QUESTION 24. Can $g(\xi)$ be effectively computed for any class of contact structures? If so, is it an effective invariant of the contact structure? Is it related to any properties of $\xi$ ?

QUESTION 25. What is the minimum of $g(\xi)$ over all tight structures (or over all universally tight structures, etc.) on $M$ ?

We also ask:

QUESTION 26. Can the contact homology or symplectic field theory [25] of a contact structure be computed in terms of an adapted open book decomposition?

Since a contact structure can be reconstructed from an adapted open book decomposition, it is clear that contact homology can be computed from the open book data. We would like to see an effective algorithm that takes dynamical information about the monodromy (and/or topological information about the binding) of the open book decomposition and outputs the contact homology.

Open book decompositions have been used to study the topology of 3-manifolds for quite some time now. So we end this section with a very open ended problem.

Problem 27. Find purely topological applications of Giroux's correspondence between convex contact structures and open book decompositions.

Though answering this problem might seem overly optimistic, Giroux and Goodman [45] have been able to answer some longstanding questions about fibered links using this machinery. 

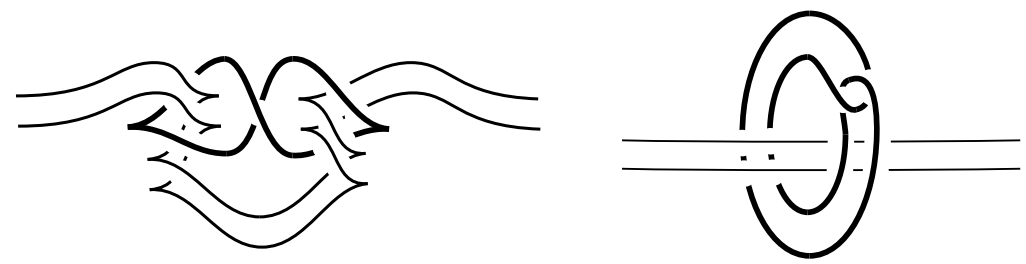

FiguRE 1. Legendrian surgery on the lighter curves on the left can yield a virtually overtwisted contact structure. The darker curve bounds an immersed disk, as shown on the right, with respect to which its twisting number is 0 .

\section{Contactomorphisms}

There is very little known concerning the group of contactomorphisms of a given contact structure.

Problem 28. Given a contact structure $\xi$ on $M$, understand the group Diff $(M, \xi)$ of contactomorphisms of $\xi$.

Several interesting subproblems are:

Problem 29. Compute $\pi_{0}(\operatorname{Diff}(M, \xi))$.

Problem 30. Study the inclusion map from Diff $(M, \xi)$ to Diff ${ }^{+}(M)$, the group of orientation-preserving diffeomorphisms.

For $S^{3}$ with the standard tight contact structure, this map is an isomorphism on the $\pi_{0}$ level. What can one say about higher homotopy groups? The inclusion is not surjective on $\pi_{0}$ for tight contact structures on $T^{3}$, since a preferred direction of "twisting" is picked out by a contact structure. We also know that for $\left(T^{3}, \xi_{n}\right)$, the kernel of the inclusion on $\pi_{0}$ contains at least $n$ elements.

Problem 31. Compute Diff $(M, \xi)$ when $\xi$ is overtwisted.

Eliashberg's work in [17] should be very helpful here, but there are technical hypotheses in most of the theorems in $[\mathbf{1 7}]$ which make it difficult to address this problem directly. In [16], it has been shown that $\pi_{0}$ for an overtwisted contact structure contains at least two elements. In order to address Problem 31 using [17], one might first want to consider the following.

QUESTION 32. Does there exist an overtwisted contact structure with two overtwisted disks which are not contact isotopic?

Note that Eliashberg's theorem does not state that homotopy classes of plane fields are in one-to-one correspondence with overtwisted contact structures; this is only true at the $\pi_{0}$ level.

\section{Miscellaneous}

Gompf [46] has shown that if a contact structure is obtained from Legendrian surgery on a Legendrian knot as in Figure 1, and the immersed disk on the left of Figure 1 lifts to an embedded disk in a finite cover, then the contact structure is virtually overtwisted. The reason is that there is a Hopf-like unknot bounding an immersed overtwisted disk that can be unwound in a cover. 
QUESTION 33. Can we always find such an immersed disk in a virtually overtwisted contact structure?

If the virtually overtwisted phenomenon could be sufficiently well understood, one could try to address the following question.

QUESTION 34. Given a virtually overtwisted contact structure, is there some procedure to find an associated universally tight structure?

Said another way, are all contact structures obtained from universally tight contact structures via some surgery-type construction?

When we perform a Lutz twist on a contact structure, a tube of overtwisted disks is produced. This raises the natural question:

QUESTION 35. Can an overtwisted disk always be completed to an overtwisted tube?

This is unlikely, given the fact that $-P$ does not support a tight contact structure. However, perhaps the putative overtwisted tube is only immersed, while each overtwisted disk is actually embedded.

QUESTION 36. If a contact structure has an overtwisted tube, is there an unLutz-twist which yields a tight structure?

\section{Legendrian Knots}

Please refer to the article by Etnyre [31], elsewhere in this volume, for introductory definitions concerning Legendrian and transverse knots. Unless otherwise stated, the ambient contact manifold is assumed to be $\left(S^{3}, \xi_{s t d}\right)$. Sections 6 through 8 deal with the problem of classifying Legendrian and transverse knots; Sections 9 and 10 address applications of these knots to contact geometry and topology.

\section{Examples}

If $\mathcal{K}$ is a topological knot type in any contact 3-manifold, let $\mathcal{L}(\mathcal{K})$ denote the set of Legendrian knots of type $\mathcal{K}$. The main problem in Legendrian knot theory is the following.

Problem 37. Given $\mathcal{K}$, classify the Legendrian knots in $\mathcal{L}(\mathcal{K})$ up to Legendrian isotopy.

In the case $\left(S^{3}, \xi_{s t d}\right)$, recall that there are two "classical invariants" of Legendrian knots, tb and $r$. A knot type is called Legendrian simple if two Legendrian knots of this type with equal classical invariants must be Legendrian isotopic. For Legendrian simple knots, classification is concluded by calculating precisely which values of $(\mathrm{tb}, r)$ can be achieved.

All of the prime knot types which we can presently classify are Legendrian simple:

(1) the unknot in any tight contact structure [24]

(2) torus knots in any tight contact structure [33]

(3) the figure eight knot in $\left(S^{3}, \xi_{\text {std }}\right)[33]$. 


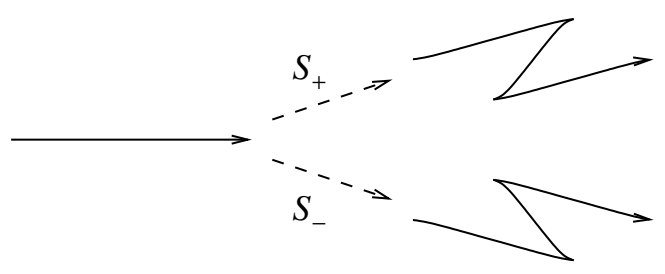

Figure 2. Stabilization of a Legendrian knot replaces a segment of the knot's front $(y z)$ projection with a zigzag as shown. There are two different stabilizations $S_{ \pm}$, which change $r$ by \pm 1 .

We can also classify Legendrian knots in a non-prime knot type if we know the classification for the prime summands [35]; see Section 7. Thus, in our discussions below, we will usually restrict attention to prime knot types. In addition, for all of the above knot types, any Legendrian knot which does not maximize tb in its knot type can be obtained from a maximal-tb knot through the operation of stabilization, which adds zigzags to the front $(y z)$ projection of the knot; see Figure 2. (It is straightforward to show that the stabilizations $S_{ \pm}$are well-defined operations up to Legendrian isotopy.) The classification results of $[\mathbf{3 3}, \mathbf{3 5}]$, and probably future classifications, rely on the powerful theory of convex surfaces developed by Giroux; see Etnyre's article [31].

Not all knot types are Legendrian simple, as we will see in Section 8. For instance, the $5_{2}$ knot shown in Figure 4 (a few pages hence) is not Legendrian simple. (Here we use the usual knot terminology from, e.g., [71].)

We digress for a moment to discuss the situation of transverse knots. Call a knot type transversely simple if all transverse knots in this knot type are completely determined by their self-linking number. Using characteristic foliation techniques, Eliashberg [21] showed that the unknot is transversely simple; similarly, Etnyre $[\mathbf{2 9}]$ and Etnyre-Honda [33] have shown that torus knots and the figure eight knot are transversely simple. Through a completely different approach, using braid foliations, Birman and Wrinkle [5] have proved that all so-called exchange reducible knots are transversely simple; by work of Menasco [64], these include all iterated torus knots. Birman and Menasco [4] have adapted difficult results in braid theory to construct families of knots which are not transversely simple.

Further results about transverse knots seem likely to arise from their connection with Legendrian knots. Given an oriented Legendrian knot $K$, we obtain two transverse knots $K_{+}$and $K_{-}$by pushing the knot off of itself in a direction tangent to the contact field so that the orientation induced on $K_{+}$and $K_{-}$as transverse knots agrees/disagrees with the orientation on $K$. (Note there is some discrepancy in the literature as to the sign convention. The one given here seems natural from many perspectives, but the other choice is also frequently used, e.g. in [3].) Conversely, any transverse knot is transverse isotopic to a knot thus obtained. Now if we denote by $S_{ \pm}$the operations of positive and negative stabilization on Legendrian knots, then two transverse knots $\left(K_{1}\right)_{+},\left(K_{2}\right)_{+}$are transverse isotopic if and only if the Legendrian knots $K_{1}, K_{2}$ are stably isotopic, i.e., $S_{-}^{n} K_{1}, S_{-}^{n} K_{2}$ are isotopic for some $n \geq 0$; see, e.g., $[\mathbf{2 7}, \mathbf{3 3}]$. It follows that classifying transverse knots up to transverse isotopy is equivalent to classifying Legendrian knots up to stable isotopy. 
In trying to attack the main Legendrian problem, we would like to mention some particularly important subproblems.

Problem 38. Classify Legendrian knots in $\mathcal{L}(\mathcal{K})$, where

(1) $\mathcal{K}$ is a fibered knot;

(2) $\mathcal{K}$ is a hyperbolic knot;

$(3) \mathcal{K}$ is not Legendrian simple;

(4) $\mathcal{K}$ is any knot type in any overtwisted contact structure.

Here are some remarks about these subproblems.

Problem 38 (1): It is currently possible (though not likely) that prime fibered knots are always Legendrian simple (for non-prime knots see below). We note that the only fibered knots of genus 1 are the figure eight knot and the right- and lefthanded trefoil knots, and Legendrian knots in all of these knot types have been classified. The next step is to look at genus 2 fibered knots. It is probable that the techniques in [33] can be used in this situation (at least sometimes), but it seems that some new ideas will be needed as the genus of the knot grows.

Problem 38 (2): We currently know very little about the Legendrian classification of hyperbolic knots; so far, we only understand Legendrian figure eight knots. As mentioned in Section 1, it would be very helpful to understand interactions between hyperbolic and contact geometry, and some clues to this interaction could come from understanding some more Legendrian knots in hyperbolic knot types. In particular, it would be great to have a Legendrian classification in a non-fibered hyperbolic knot type. However, it might be easier to try knot types that are both hyperbolic and fibered. W. Kazez has suggested looking at the knot $8_{20}$; this is a fibered hyperbolic knot whose holonomy seems to be interesting.

Problem 38 (3): There are currently no prime knot types $\mathcal{K}$ that are not Legendrian simple for which we can classify $\mathcal{L}(\mathcal{K})$. The most obvious one to consider is the $5_{2}$ knot type.

CONJECTURE 39. There are exactly two Legendrian knots realizing the $5_{2}$ knot type with $\mathrm{tb}=1$ and $r=0$. All other Legendrian knots realizing $5_{2}$ are stabilizations of these, and are determined by their tb and $r$.

Problem 38 (4): It is a surprising fact that much progress has been made towards classifying Legendrian knots in tight contact structures, but little is known about Legendrian knots in overtwisted contact structures. Call a Legendrian knot $K$ in an overtwisted contact structure loose if the contact structure on the complement of $L$ is overtwisted. It seems that most Legendrian knots in overtwisted contact structures are loose, but there are also non-loose Legendrian knots [16]. If two loose Legendrian knots have the same knot type and tb and $r$, then they are Legendrian isotopic $[\mathbf{1 6}, \mathbf{2 4}]$. Thus it would be interesting to know:

QUESTION 40. For what contact manifolds and knot types $\mathcal{K}$ are all Legendrian knots in $\mathcal{L}(\mathcal{K})$ loose?

For example, let $\xi^{\prime}$ be the contact structure on $S^{3}$ obtained from the tight contact structure by applying one Lutz twist.

Conjecture 41. If $\xi$ is any overtwisted contact structure on $S^{3}$ besides $\xi^{\prime}$, then all Legendrian unknots are loose. 
Conjecture 42. Any Legendrian unknot in $\xi^{\prime}$ with tb $\neq 1$ or $r \neq 0$ is loose, and there are exactly two Legendrian unknots with tb $=1$ and $r=0$, one loose, one not.

As a final remark, we note that any Legendrian knot that violates the Bennequin inequality is automatically loose.

\section{Structure Results}

A prototypical structure result for Legendrian knots concerns connected sums. Given two Legendrian knots $L_{1} \in \mathcal{L}\left(\mathcal{K}_{1}\right)$ and $L_{2} \in \mathcal{L}\left(\mathcal{K}_{2}\right)$, we can form their connected sum $L_{1} \# L_{2}$ in a standard way (see, e.g., [12]). In [35] it is shown that connected sum of Legendrian knots gives a bijection

$$
\left(\frac{\mathcal{L}\left(\mathcal{K}_{1}\right) \times \mathcal{L}\left(\mathcal{K}_{2}\right)}{\sim}\right) \longrightarrow \mathcal{L}\left(\mathcal{K}_{1} \# \mathcal{K}_{2}\right)
$$

where the equivalence relation $\sim$ is of two types:

(1) $\left(L_{1}, S_{ \pm}\left(L_{2}\right)\right) \sim\left(S_{ \pm}\left(L_{1}\right), L_{2}\right)$,

(2) $\left(L_{1}, L_{2}\right) \sim\left(L_{2}, L_{1}\right)$ if $\mathcal{K}_{1}=\mathcal{K}_{2}$.

Thus we can classify $\mathcal{L}\left(\mathcal{K}_{1} \# \mathcal{K}_{2}\right)$ in terms of $\mathcal{L}\left(\mathcal{K}_{1}\right)$ and $\mathcal{L}\left(\mathcal{K}_{2}\right)$. Using this result and the classification of Legendrian negative torus knots, one may easily find nonLegendrian-simple fibered knot types $\mathcal{K}$ for which we can completely classify $\mathcal{L}(\mathcal{K})$.

Virtually nothing is known about the structure of $\mathcal{L}(\mathcal{K})$ for general knot types $\mathcal{K}$. One important notion here is destabilizing a Legendrian knot, i.e., isotoping it to become a stabilization of another Legendrian knot, and then removing the stabilization; this raises tb by 1 .

Question 43. Do all Legendrian knots destabilize until they reach the maximal possible tb for their knot type?

It seems unlikely that the answer to this question is yes in general, but it is yes for all knot types for which the question has been answered [33]. Question 43 is important since the method used in all current classification results is to show Legendrian knots destabilize to maximal tb knots, and then to classify these. This scheme would have real difficulties if the answer to the following question were yes.

QUESTION 44. Is there a knot type with Legendrian representatives that do not destabilize but have arbitrarily negative tb?

Give a knot type $\mathcal{K}$, let $\mathcal{K}_{(p, q)}$ be the $(p, q)$-cable of $\mathcal{K}$; that is, a representative of $\mathcal{K}_{(p, q)}$ sits on the boundary of a tubular neighborhood of a representative of $\mathcal{K}$ as a $(p, q)$ curve. The techniques in [33] should allow one to address the following.

Problem 45. Classify Legendrian knots in $\mathcal{L}\left(\mathcal{K}_{(p, q)}\right)$ in terms of $\mathcal{L}(\mathcal{K})$.

In addition to connected sum and cabling, there are many other operations one can perform on knots.

QUESTION 46. What can one say about Legendrian knots that are in a knot type obtained from

(1) Whitehead doubling or some other satellite construction?

(2) Murasugi sums?

(3) your other favorite topological abuse of knots? 


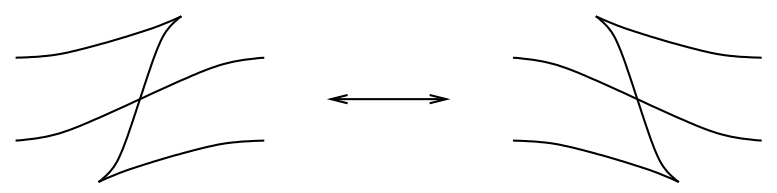

FiguRE 3. Basic move preserving tb and topological class, but not necessarily Legendrian isotopy class.

For background on Legendrian satellites, see, e.g., [68]. It is plausible that Whitehead doubling two non-isotopic Legendrian knots leads to non-isotopic Legendrian knots; if the original knots have the same tb, then the doubles have the same tb and $r$. Examples similar to the non-isotopic knots of Figure 4 can be constructed by doubling unknots with the same tb but different $r$.

Here are some other interesting structural questions.

QUESTION 47. How are the Legendrian knots in a knot type affected by crossing changes of the knot type?

For example, is there a formula for how the maximal value of tb changes under crossing changes?

QUESTION 48. If $K_{1}$ and $K_{2}$ are topologically isotopic with the same tb, is there a set of local moves which, along with Legendrian isotopy, will send $K_{1}$ to $K_{2}$ ?

A basic move suggested by $\mathrm{L}$. Ng is given in Figure 3. This move seems to suffice in many examples. However, there are examples in which this is not sufficient, as one can show using the characteristic algebra (see Section 8).

A result of Fuchs and Tabachnikov [39] states that two topologically isotopic Legendrian knots $K_{1}$ and $K_{2}$, with identical classical invariants, must have $S_{+}^{n_{1}} S_{-}^{n_{2}} K_{1}$ and $S_{+}^{n_{1}} S_{-}^{n_{2}} K_{2}$ Legendrian isotopic for some $n_{1}, n_{2} \geq 0$. (Note that we need both positive and negative stabilizations.) By considering Legendrian knots that are topologically connected sums of negative torus knots, one sees that the number of necessary stabilizations can be arbitrarily large [35], but one can ask:

QUESTION 49. Are there bounds on the number of stabilizations necessary to make two Legendrian knots isotopic, in terms of the topology of the knot type?

\section{Legendrian invariants and contact homology}

The first example of a knot type which is not Legendrian simple was produced independently by Chekanov [12] and Eliashberg and Hofer [23]. As mentioned before, the knot type is $5_{2}$; the two non-isotopic Legendrian $5_{2}$ knots with equal classical invariants are shown in Figure 4.

The method used to distinguish between the two $5_{2}$ knots is a new, powerful invariant of Legendrian knots, motivated by the development of contact homology [23], which applies Gromov's holomorphic-curve techniques to contact manifolds. This invariant takes the form of a differential graded algebra (DGA), a free noncommutative unital algebra with a grading and differential; the homology of the algebra is precisely the contact homology of $S^{3}$ relative to the Legendrian knot (see $[36])$. 

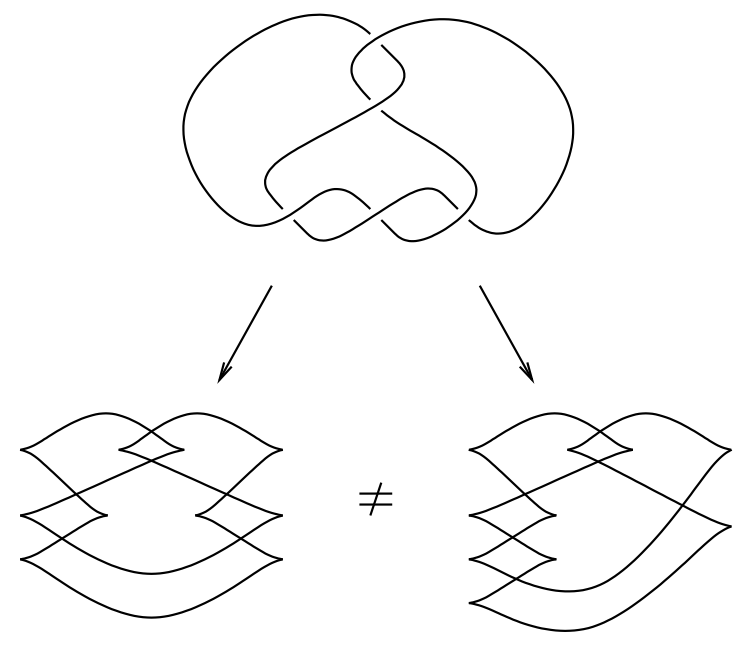

Figure 4. Example, due to Chekanov and Eliashberg-Hofer, of two Legendrian knots, both of topological type $5_{2}$ and with $t b=1$ and $r=0$, which are not Legendrian isotopic.

Although contact homology is difficult to compute in general, this relative contact homology has a simple combinatorial formulation due to Chekanov [12]; in addition, it can be treated completely combinatorially, without reference to the general theory of contact homology. Chekanov's definition allows us to write down the DGA invariant explicitly, given the knot diagram of a Legendrian knot. Two Legendrian knots are then isotopic only if they have equivalent DGAs, under an algebraic equivalence called "stable tame isomorphism." The formulation of the DGA has subsequently been refined in $[\mathbf{3 6}, \mathbf{6 5}, \mathbf{6 7}]$.

In practice, because of the difficulty of manipulating the DGA directly, applications have used simpler invariants derived from the DGA. The easiest are Poincaré-type polynomials which calculate the graded dimension of linearizations of the DGA. Chekanov used these polynomials to show that the knots in Figure 4 are not Legendrian isotopic. Along the same lines, it can be shown $[\mathbf{1 2}, \mathbf{2 7}]$ that there exist knot types and tb and $r$ for which there are arbitrarily many non-isotopic Legendrian representatives.

A more involved DGA-derived invariant is the characteristic algebra [67]. This can be used to answer (in the negative) the Legendrian mirror question of Fuchs and Tabachnikov [39], which asks whether a Legendrian knot with $r=0$ must necessarily be Legendrian isotopic to its mirror, its image under the contactomorphism $(x, y, z) \mapsto(x,-y,-z)$. Further applications of the characteristic algebra are given in [67], including knots distinguished without using any sort of grading. It seems possible that the characteristic algebra is also linked to topology.

Conjecture $50(\mathrm{Ng})$. The abelianized characteristic algebra is a topological invariant. More precisely, it depends only on $\mathcal{K}$ and tb.

QUeSTION 51. Fully understand the space of DGAs modulo stable tame isomorphism. In particular, are there other useful invariants which can be derived from the DGA? 
Along these lines, it would be particularly nice to answer:

QUESTION 52. If two DGAs have the same homology, are they stable tame isomorphic?

Chekanov and Pushkar [13] have discovered another non-classical invariant of Legendrian knots, using admissible decompositions of fronts. No applications of this invariant are currently known, besides examples in which the Poincaré-polynomial technique can already be used. In fact, the following seems possible; see [38] for a result in this direction.

Conjecture 53. The Chekanov-Pushkar invariant can be deduced from Poincaré polynomials for the corresponding DGA.

Analogues of the DGA theory can be derived for contact manifolds other than $\left(S^{3}, \xi_{s t d}\right)$.

Problem 54. Construct combinatorial relative contact homology theories for Legendrian knots in other contact manifolds.

This has been done for the solid torus $S^{1} \times \mathbb{R}^{2}$ with the standard tight contact structure by Ng and Traynor, and, with more difficulty, for circle bundles over closed orientable surfaces with a contact structure transverse to the fibers [73]. We note that for $S^{1} \times \mathbb{R}^{2}$, Traynor [77] has defined another non-classical invariant for a particular class of links, using generating functions and algebraic topology; mysteriously, for these links, all current calculations indicate that this invariant coincides with a link-graded Poincaré polynomial derived from the DGA.

We now return to $\left(S^{3}, \xi_{s t d}\right)$. One major remaining difficulty with the DGA invariant is that it vanishes for stabilized Legendrian knots.

Problem 55. Are there useful contact-homology-type invariants for stabilizations?

This problem is of particular importance in trying to understand transverse knots; see Section 6.

One possible approach to understanding invariants for stabilizations is to use satellite constructions, which in many cases produce knots with nontrivial DGAs. The simplest possible satellite, the Whitehead double, yields no interesting invariants for stabilizations [68], but more complicated satellites may encode useful information.

Another possible approach is to apply the symplectic field theory of EliashbergGivental-Hofer [25], which generalizes contact homology, to Legendrian knots.

Problem 56. Combinatorially understand the full symplectic field theory for Legendrian knots.

The algebraic structure behind absolute symplectic field theory is somewhat well understood [25], but this is far from the case for the relative symplectic field theory needed for the Legendrian case. Here, the algebraic formalism has resisted several attempts at a reasonable definition.

Given a Legendrian knot $K$ in $(M, \xi)$, there is a natural $(\operatorname{tb}(K)-1)$ surgery on $K$, called Legendrian surgery, which produces another contact manifold; see $[18,46,78]$ for background. 
Problem 57. Let $K$ be a Legendrian knot inside $\left(S^{3}, \xi_{\text {std }}\right)$, and let $(M, \xi)$ be the Stein fillable contact structure obtained by performing Legendrian surgery on $K$. Is there a way to calculate the contact homology of $(M, \xi)$ from the DGA invariant associated to $K$ ?

Though finding such a relation would be great, it seems unlikely that one can determine the contact homology of $(M, \xi)$ merely from the DGA of $K$; however, one can almost certainly compute the contact homology of $(M, \xi)$ from the information which would define the symplectic field theory of $K$. Thus it is probably more realistic to try to solve Problem 56 first before attacking this problem. As a possibly simpler first step, we ask the following.

Question 58. If $(M, \xi)$ in Problem 57 is obtained from a stabilized Legendrian knot, does the contact homology of $(M, \xi)$ have a simple form? (Is is almost trivial? Does it equal the base field?)

\section{Relation to contact geometry}

We now turn from intrinsic properties of Legendrian knots to applications of Legendrian knots to contact geometry. Bennequin $[\mathbf{3}]$ famously established the existence of an exotic contact $\mathbb{R}^{3}$ by studying transverse unknots, and Legendrian knots have played a similar role in distinguishing between contact structures on the 3 -torus [56] and homology spheres [1]. In each case, one investigates what sorts of Legendrian (or transverse) knots can be carried by the contact structure.

Problem 59. Distinguish other contact structures using Legendrian knots.

A more precise version of this problem might be given by the following question.

QUESTION 60. Can tight contact structures $\xi_{1}$ and $\xi_{2}$ on the same manifold $M$ always be distinguished by finding a Legendrian knot of a certain tb (or twisting number) and $r$ which exists in one, but not in the other?

For example, the tight contact manifolds $\left(T^{3}=\mathbb{R}^{3} / \mathbb{Z}^{3}, \xi_{n}\right), n \in \mathbb{Z}^{+}$, given by the 1 forms $\alpha_{n}=\sin (2 \pi n z) d x+\cos (2 \pi n z) d y$, are distinguished by the maximal twisting number among curves isotopic to $x=y=$ const [56]. More generally, tight contact structures on $T^{2}$ bundles over $S^{1}$ are determined by their homotopy class of plane field and by information about Legendrian knots [51].

It would be interesting to see if the Lisca-Matić examples [60] of contact structures can also be distinguished in this way. These are tight structures on the Brieskorn sphere $\Sigma(p, q, p q n-1)$, obtained by surgery on distinct Legendrian knots. Via Seiberg-Witten theory, the contact structures are distinguished by the Chern classes of Stein 4-manifolds which bound $\Sigma(p, q, p q n-1)$. Could one use Legendrian knot theory instead of Seiberg-Witten theory to distinguish these examples?

We next address the effect of Legendrian surgery on contact structures. It is known [18] that Legendrian surgery on a knot in a fillable contact structure produces another fillable structure.

Question 61. Does Legendrian surgery preserve tightness?

If we allow manifolds with boundary, the answer is no [52] (see also [10]). Nothing is known for the closed case. 
Question 62. Consider two Legendrian knots $K_{1}, K_{2}$ with the same tb and $r$ which are not isotopic. Let $\left(M, \xi_{1}\right)$ and $\left(M, \xi_{2}\right)$ be the contact manifolds obtained by performing Legendrian surgery on $K_{1}$ and $K_{2}$, respectively. Are $\xi_{1}$ and $\xi_{2}$ necessarily isotopic?

One might like to begin working on this problem by considering the maximal tb examples of the $5_{2}$ knot.

Here are some final questions.

Question 63. Let $K$ be a maximal tb Legendrian knot in $\left(S^{3}, \xi_{s t d}\right)$. Is $\left(S^{3} \backslash\right.$ $\left.K,\left.\xi_{\text {std }}\right|_{S^{3} \backslash K}\right)$ universally tight?

QUESTION 64. If $K$ has non-maximal tb but is not a stabilization (cf. Question 43), is $\left(S^{3} \backslash K,\left.\xi_{s t d}\right|_{S^{3} \backslash K}\right)$ universally tight?

Though it is possible for the answers of these questions to be yes, that would be quite amazing. More realistically, we can ask:

QUESTION 65. If $K$ is a maximal tb Legendrian knot in $\left(S^{3}, \xi_{\text {std }}\right)$, is the $\mathbb{Z}$ cover of $S^{3} \backslash K$ tight?

Understanding these questions could be helpful in addressing Question 61, as well as providing a connection to the topology of the knot.

\section{Relation to topology}

Legendrian knot theory has deep connections with topology as well as with contact geometry, principally through the information encoded in the ThurstonBennequin invariant of a Legendrian knot in $\left(S^{3}, \xi_{\text {std }}\right)$. For a knot type $\mathcal{K}$, let $\overline{\operatorname{tb}}(\mathcal{K})$ denote the maximal tb over all Legendrian knots of type $\mathcal{K}$. This number is finite; see the next paragraph.

Bennequin's proof of the existence of an exotic contact structure on $S^{3}$ reduces, in modern terminology, to establishing that the unknot in $\left(S^{3}, \xi_{\text {std }}\right)$ satisfies $\overline{\mathrm{tb}}=$ -1 . His method extends to an upper bound on $\overline{\operatorname{tb}}(\mathcal{K})$ for all $\mathcal{K}$, in terms of the genus of $\mathcal{K}$. Since then, upper bounds for $\overline{\mathrm{tb}}$ have been obtained in terms of the slice genus, HOMFLY polynomial, and Kauffman polynomial; see [37] for a survey of results. In particular, Rudolph [72] (q.v. $[\mathbf{5 7}, \mathbf{6 0}]$ ) has shown that if $\overline{\operatorname{tb}}(\mathcal{K}) \geq 0$, then $\mathcal{K}$ is not slice.

In this vein, consider the smooth knot concordance group $\mathcal{C}$; this is a direct sum of countably many $\mathbb{Z}, \mathbb{Z} / 2 \mathbb{Z}$, and possibly $\mathbb{Z} / 4 \mathbb{Z}$ factors. It is currently unknown whether there are any, or even countably many, $\mathbb{Z} / 4 \mathbb{Z}$ factors. One can see (at least some of) the $\mathbb{Z} / 2 \mathbb{Z}$ factors from the fact that amphichiral knots have order 2 . The result of Rudolph allows us to see some of the $\mathbb{Z}$ factors as well, through Legendrian knot theory; if $\overline{\operatorname{tb}}(\mathcal{K}) \geq 0$, then $\mathcal{K}$ is of infinite order in $\mathcal{C}$.

QUESTION 66. Can we use Legendrian knots to improve or reprove other results concerning slice knots?

See, e.g., $[\mathbf{7}, \mathbf{6 1}]$. One might be able to prove results such as the following.

ConjeCture 67 (Etnyre). A knot is either of order 2, or concordant to a knot for which either it or its mirror has $\overline{\mathrm{tb}} \geq 0$.

We can consider topological properties besides sliceness, as well. 
Problem 68. Relate Legendrian knot invariants to the Alexander module.

Questions 63, 64, and 65 are relevant to this problem. We also have the following related problem; see [14] for a start.

PROBLEM 69. Find relationships between Legendrian knot invariants and the Alexander norm or Thurston norm.

It would also be illuminating to consider:

Problem 70. Understand more deeply the relation between $\mathcal{L}(\mathcal{K})$ and $\overline{\mathrm{tb}}(\mathcal{K})$ on the one hand, and the HOMFLY and Kauffman polynomials on the other.

According to Fuchs-Tabachnikov, there are no finite-type invariants of Legendrian knots besides tb, $r$, and standard topological finite-type invariants. Can one extract non-finite-type topological knot invariants by looking at $\mathcal{L}(\mathcal{K})$ ? In particular:

\section{Question 71. Is $\overline{\mathrm{tb}}$ a non-finite-type invariant?}

One possible motivation for applying Legendrian knot theory to topological knot theory is that Legendrian knots are very sensitive to mirroring; e.g., $\mathcal{K}$ and its mirror can have vastly different $\overline{\mathrm{tb}}$.

Finally, showing that a Legendrian knot is determined by its complement is somewhat easy, using Eliashberg's classification of tight contact structures on $\left(S^{3}, \xi_{\text {std }}\right)$. However, the topological knot complement problem requires the ingenuity of Gordon and Luecke [47].

PROBLEM 72. Use Legendrian knot theory to reprove the topological knot complement result.

\section{References}

[1] S. Akbulut and R. Matveev, A note on contact structures, Pacific J. Math. 182 (1998), 201-204.

[2] S. Akbulut and B. Ozbagci, Lefschetz fibrations on compact Stein surfaces, Geom. Topol. 5 (2001), 319-334.

[3] D. Bennequin, Entrelacements et équations de Pfaff, Astérisque 107-108 (1983), 87-162.

[4] J. Birman and W. Menasco, Stabilization in the braid groups, preprint, math.GT/0203227.

[5] J. Birman and N. Wrinkle, On transversally simple knots, J. Diff. Geom. 55 (2000), 325-354.

[6] D. Calegari and N. Dunfield, Criteria for non existence of taut foliations, in preparation.

[7] T. Cochran and K. Orr, Not all links are concordant to boundary links. Ann. of Math. (2) 138 (1993), 519-554.

[8] V. Colin, Chirurgies d'indice un et isotopies de sphères dans les variétés de contact tendues, C. R. Acad. Sci. Paris Sér. I Math. 324 (1997), 659-663.

[9] V. Colin, Une infinité de structures de contact tendues sur les variétés torö̈dales, Comment. Math. Helv. 76 (2001), 353-372.

[10] V. Colin, Chirurgies de Dehn admissibles dans les variétés de contact tendues, Ann. Inst. Fourier (Grenoble) 51 (2001), 1419-1435.

[11] V. Colin, Sur la torsion des structures de contact tendues, Ann. Sci. École Norm. Sup. (4) 34 (2001), 267-286.

[12] Y. Chekanov, Differential algebra of Legendrian links, Invent. Math., to appear.

[13] Y. Chekanov and P. Pushkar, Arnold's four cusp conjecture and invariants of Legendrian knots, in preparation.

[14] O. Dasbach and B. Mangum, On McMullen's and other inequalities for the Thurston norm of link complements, Algebr. Geom. Topol. 1 (2001), 321-347.

[15] F. Ding and H. Geiges, Symplectic fillability of tight contact structures on torus bundles, Algebr. Geom. Topol. 1 (2001), 153-172. 
[16] K. Dymara, Legendrian knots in overtwisted contact structures on $S^{3}$, Ann. Global Anal. Geom. 19 (2001), 293-305.

[17] Y. Eliashberg, Classification of overtwisted contact structures on 3-manifolds, Invent. Math. 98 (1989), 623-637.

[18] Y. Eliashberg, Topological characterization of Stein manifolds of dimension > 2, Internat. J. Math. 1 (1990), 29-46.

[19] Y. Eliashberg, Filling by holomorphic discs and its applications, Geometry of LowDimensional Manifolds, Vol. II, Donaldson and Thomas, eds. (Cambridge University Press, Cambridge, 1990).

[20] Y. Eliashberg, Contact 3-manifolds twenty years since J. Martinet's work, Ann. Inst. Fourier (Grenoble) 42 (1992), 165-192.

[21] Y. Eliashberg, Legendrian and transversal knots in tight contact 3-manifolds, Topological methods in modern mathematics (Stony Brook, NY, 1991), 171-193 (Publish or Perish, Houston, 1993).

[22] Y. Eliashberg, Unique holomorphically fillable contact structure on the 3-torus, Internat. Math. Res. Notices 1996, 77-82.

[23] Y. Eliashberg, Invariants in contact topology, Doc. Math. J. DMV Extra Volume ICM 1998 (electronic), 327-338.

[24] Y. Eliashberg and M. Fraser, Classification of topologically trivial Legendrian knots, in Geometry, topology, and dynamics (Montreal, PQ, 1995), CRM Proc. Lecture Notes, 15 (Amer. Math. Soc., Providence, 1998).

[25] Y. Eliashberg, A. Givental, and H. Hofer, Introduction to symplectic field theory, GAFA 2000 (Tel Aviv, 1999), Geom. Funct. Anal. 2000, Special Volume, Part II, 560-673.

[26] Y. Eliashberg and W. Thurston, Confoliations (Amer. Math. Soc., Providence, 1998).

[27] J. Epstein, D. Fuchs, and M. Meyer, Chekanov-Eliashberg invariants and transverse approximations of Legendrian knots, Pacific J. Math. 201 (2001), 89-106.

[28] J. Etnyre, Symplectic convexity in low-dimensional topology, Top. Appl. 88 (1998), 3-25.

[29] J. Etnyre, Transversal torus knots, Geom. Topol. 3 (1999), 253-268.

[30] J. Etnyre, Tight contact structures on lens spaces, Commun. Contemp. Math. 2 (2000), $559-577$.

[31] J. Etnyre, Introductory lectures on contact geometry, elsewhere in these proceedings.

[32] J. Etnyre and K. Honda, On the non-existence of tight contact structures, Ann. of Math. (2) 153 (2001), 749-766.

[33] J. Etnyre and K. Honda, Knots and contact geometry I: torus knots and figure eight, $J$. Sympl. Geom. 1 (2001), 63-120.

[34] J. Etnyre and K. Honda, Tight contact structures with no symplectic fillings, Invent. Math. 148 (2002), 609-626.

[35] J. Etnyre and K. Honda, Konts and contact geometry II: connected sums, preprint, math.SG/0205310.

[36] J. Etnyre, L. Ng, and J. Sabloff, Coherent orientations and invariants of Legendrian knots, J. Sympl. Geom., to appear; also available at math.SG/0101145.

[37] E. Ferrand, On Legendrian knots and polynomial invariants, Proc. Amer. Math. Soc. 130 (2002), 1169-1176.

[38] D. Fuchs, Chekanov-Eliashberg invariants of Legendrian knots: existence of augmentations, preprint.

[39] D. Fuchs and S. Tabachnikov, Invariants of Legendrian and transverse knots in the standard contact space, Topology 36 (1997), 1025-1054.

[40] D. Gabai, Foliations and the topology of 3-manifolds, J. Diff. Geom. 18 (1983), 115-503.

[41] P. Ghiggini, S. Schönenburger, On the classification of tight contact structures, elsewhere in these proceedings.

[42] E. Giroux, Convexité en topologie de contact, Comment. Math. Helv. 66 (1991), 637-677.

[43] E. Giroux, Structures de contact en dimension trois et bifurcations des feuilletages de surfaces, Invent. Math. 141 (2000), 615-689.

[44] E. Giroux, Structures de contact sur les variétés fibrées en cercles au-dessus d'une surface, Comment. Math. Helv. 76 (2001), 218-262.

[45] E. Giroux, Talks at Stanford, December 2000, and at the University of Georgia, May 2001.

[46] R. Gompf, Handlebody construction of Stein surfaces, Ann. of Math. (2) 148 (1998), 619693. 
[47] C. McA. Gordon and J. Luecke, Knots are determined by their complements. J. Amer. Math. Soc. 2 (1989), 371-415.

[48] M. Gromov, Pseudoholomorphic curves in symplectic manifolds, Invent. Math. 82 (1985), 307-347.

[49] J. Hempel, 3-Manifolds, Ann. of Math. Stud. 86 (Princeton University Press, Princeton, 1976).

[50] K. Honda, On the classification of tight contact structures I, Geom. Topol. 4 (2000), 309-368.

[51] K. Honda, On the classification of tight contact structures II, J. Diff. Geom. 55 (2000), 83-143.

[52] K. Honda, Gluing tight contact structures, Duke Math. J., to appear; also available as math.GT/0102029.

[53] K. Honda, W. Kazez, and G. Matić, Convex decomposition theory, Int. Math. Res. Not. 2002, 55-88.

[54] K. Honda, W. Kazez, and G. Matić, On the Gabai-Eliashberg-Thurston theorem, preprint, math.GT/0111123.

[55] K. Honda, W. Kazez, and G. Matić, Tight contact structures on fibered hyperbolic 3manifolds, preprint, math.GT/0110104.

[56] Y. Kanda, The classification of tight contact structures on the 3-torus, Comm. Anal. Geom. 5 (1997), 413-438.

[57] P. Kronheimer and T. Mrowka, Monopoles and contact structures, Invent. Math. 129 (1997), 509-525.

[58] P. Lisca, Symplectic fillings and positive scalar curvature, Geom. Topol. 2 (1998), 103-116.

[59] P. Lisca, On symplectic fillings of 3-manifolds, Proceedings of 6th Gökova Geometry-Topology Conference, Turkish J. Math. 23 (1999), 151-159.

[60] P. Lisca and G. Matić, Tight contact structures and Seiberg-Witten invariants, Invent. Math. 129 (1997), 509-525.

[61] C. Livingston and S. Naik, Obstructing four-torsion in the classical knot concordance group, J. Diff. Geom. 51 (1999), 1-12.

[62] A. Loi and R. Piergallini, Compact Stein surfaces with boundary as branched covers of $B^{4}$, Invent. Math. 143 (2001), 325-348.

[63] R. Matveyev, talk at Stanford, December 2000.

[64] W. Menasco, On iterated torus knots and transversal knots, Geom. Topol. 5 (2001), 651-682.

[65] K. Mishachev, Relative homotopy splitting of differential algebra of Legendrian link, preprint.

[66] A. Mori, A note on Thurston-Winkelnkemper's construction of contact forms on 3-manifolds, Osaka J. Math., 39 (2002), 1-11.

[67] L. Ng, Computable Legendrian invariants, Topology, to appear; also available as math.GT/0011265.

[68] L. Ng, The Legendrian satellite construction, preprint, math.GT/0112105.

[69] H. Ohta and K. Ono, Simple singularities and topology of symplectically filling 4-manifold, Comment. Math. Helv. 74 (1999), 575-590.

[70] R. Roberts, J. Shareshian, and M. Stein, research announcement.

[71] D. Rolfsen, Knots and links (Publish or Perish Press, Berkeley, 1976).

[72] L. Rudolph, An obstruction to sliceness via contact geometry and "classical" gauge theory, Invent. Math. 119 (1995), 155-163.

[73] J. Sabloff, Invariants of Legendrian knots in circle bundles, in preparation.

[74] I. Smith, Torus fibrations on symplectic four-manifolds, Turkish J. Math. 25 (2001), 69-95.

[75] W. Thurston, Hyperbolic structures on 3-manifolds, III: Deformations of 3-manifolds with incompressible boundary, Ann. of Math. (2), to appear; also available as math.GT/9801058.

[76] W. Thurston and H. Winkelnkemper, On the existence of contact forms, Proc. Amer. Math. Soc. 52 (1975), 345-347.

[77] L. Traynor, Generating function polynomials for legendrian links, Geom. Topol. 5 (2001), $719-760$.

[78] A. Weinstein, Contact surgery and symplectic handlebodies, Hokkaido Math. J. 20 (1991), $241-251$. 
University of Pennsylvania, Philadelphia, PA 19104

E-mail address: etnyre@math.upenn.edu

$U R L:$ http://www.math. upenn.edu/〜etnyre

American Institute of Mathematics, Palo Alto, CA 94306

E-mail address: ng@alum.mit.edu

$U R L:$ http://alum.mit.edu/www/ng 